Trauma Berufskrankh 2012 • 14[Suppl 2]:135-139 DOI 10.1007/s10039-011-1793-1

Online publiziert: 8. Oktober 2011

c) Springer-Verlag 2011
C. Jacobs · P.A. Grützner

Klinik für Unfallchirurgie und Orthopädie, BG-Unfallklinik Ludwigshafen

Was macht Sinn?

Ziel dieses Eingriffs ist die anatomische Rekonstruktion des Azetabulums, um ein stufenfreies anatomisches Alignment zu erreichen. Des Weiteren ermöglicht eine stabile Osteosynthese eine frühzeitige Mobilisation des Patienten. Die anatomische Wiederherstellung der Gelenkfläche ist die Voraussetzung für ein gutes funktionelles Behandlungsergebnis. Je nach Frakturausmaß und -lokalisation kommen limitierte, kombinierte oder erweiterte Zugänge (Maryland-Zugang), die jedoch besonders komplikationsträchtig sind, in Betracht.

\section{Postoperative Komplikationen}

Typische Komplikationen nach operativ versorgten Azetabulumfrakturen lassen sich in allgemeine und spezielle Komplikationen einteilen:

\section{Allgemeine Komplikationen}

Wundheilungsstörungen und postoperative Infektionen sind nicht selten die Folge ausgedehnter Zugänge und mehrfacher Repositionsversuche. Wundinfektionen treten bei $12-46 \%$ der Patienten auf [4], - Abb. 1 zeigt eine Wundheilungsstörung nach einem ausdehnten Maryland-Zugang.

Auch ein erhöhter Body-Mass-Index (BMI) der Patienten stellte sich mittlerweile als signifikanter Risikofaktor für eine Wundheilungsstörung heraus [4]. Nervenschäden betreffen je nach Zugang den N. ischiadicus (Kocher-Langenbeck-
Zugang) bzw. den N. femoralis (ilioinguinaler Zugang) und sind für den Patienten folgenschwere Komplikationen. Ausgedehnte Zugänge und unsaubere Präparationen führen $\mathrm{zu}$ erheblichen Weichteilschäden mit erhöhten Infektraten und Wundheilungsstörungen. Thrombembolische Ereignisse sind die Folge der Immobilisation in Kombination mit dem Beckentrauma. Nicht zuletzt deswegen sollte, wann immer möglich, eine stabile Osteosynthese das operative Behandlungsziel sein, sodass die Immobilisationszeit so kurz wie möglich gehalten werden kann. Heterotope Ossifikationen sind bekannte postoperative Probleme und können bei erheblichen Bewegungseinschränkungen Folgeeingriffe erforderlich machen.

\section{Spezielle Komplikationen}

Schwerwiegende Komplikationen nach Osteosynthesen sind v. a. Implantatversagen und -fehllagen. Meist sind operative Revisionen erforderlich, wodurch sich die Komplikationsraten erhöhen. Ein Nichterkennen von intraartikulären Implantaten führt innerhalb kürzester Zeit zu einer ausgeprägten posttraumatischen Arthrose. Daher ist eine primär korrekte Schraubenpositionierung von enormer Bedeutung und sollte intraoperativ durch Sicht oder 3D-Bildwandler kontrolliert werden.

Eine Verzögerung der osteosynthetischen Versorgung führt ebenfalls zu einem signifikanten Anstieg der Komplikationsraten [7]. 


\section{Becken, Acetabulum, hüftgelenksnahe Frakturen}

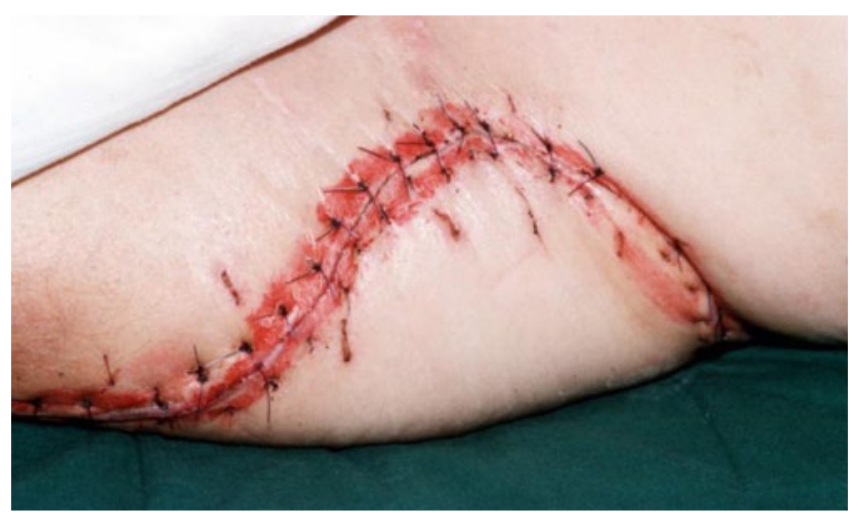

Abb. 14 Wundheilungsstörung nach Maryland-Zugang

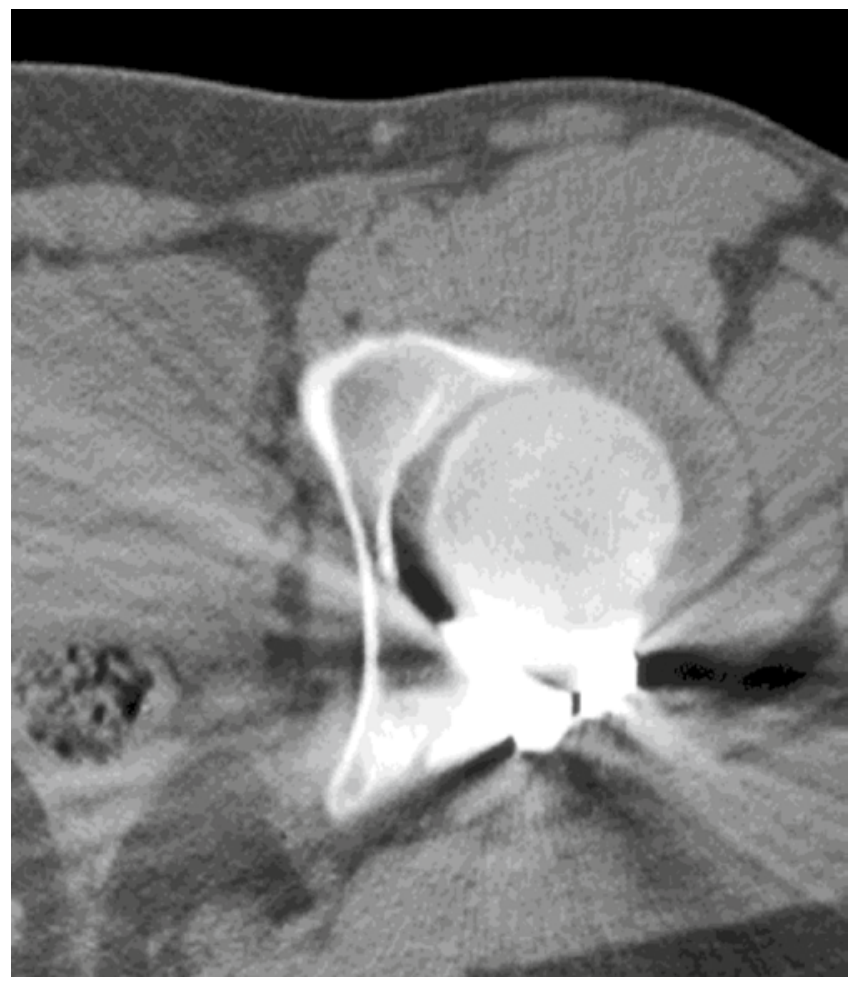

Abb. $2<$ Intraartikuläre Schraubenlage
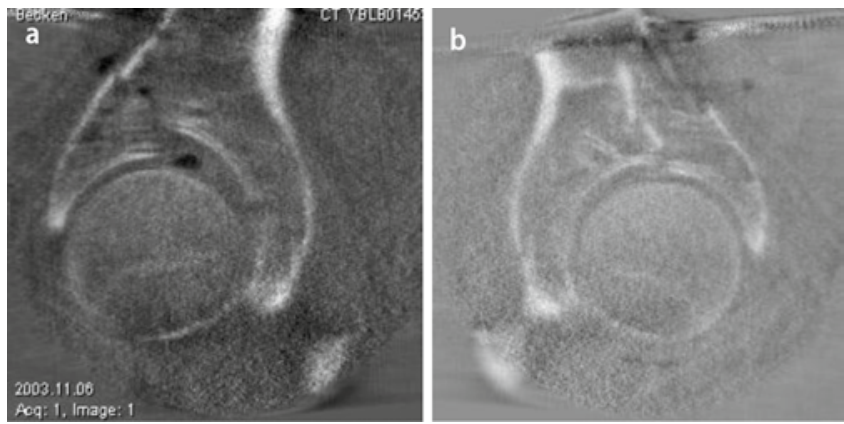

Abb. $3<$ a Zentrale Impression im Pfannendach und $\mathbf{b}$ dessen anatomische Rekonstruktion

\section{Posttraumatische Arthrose}

Ziel der Erstoperation sollte eine möglichst anatomische Rekonstruktion sein. In einer Studie von Briffa et al. [1] wurde diese bei 74\% der 161 Patienten beschrieben. Es ist allerdings anzumerken, dass diese Ergebnisse nicht durch eine post- operative CT-Untersuchung (CT: Computertomographie) bestätigt wurden. Der Durchschnittswert der erreichten anatomischen Rekonstruktionen kann daher noch niedriger sein.

Bei komplexen Frakturen kann eine stufenlose Rekonstruktion primär auch unmöglich sein, sodass eine posttraumatische Arthose vorprogrammiert ist. Insbesondere bei Frakturtypen mit mehreren Fragmenten muss daher intraoperativ ein Bildwandler, möglichst mit der Option der 3D-Darstellung, eingesetzt werden. Andererseits lässt sich auch durch eine anatomische Rekonstruktion die posttraumatische Arthrose häufig nicht verhindern. Nicht zuletzt können bereits durch den Unfall selbst eine irreversible Durchblutungsstörung des Hüftkopfs oder ein azetabulärer Knorpelschaden entstanden sein und in einer posttraumatischen Arthrose enden. Dies lässt deren hohe Inzidenz trotz anatomischer Rekonstruktion erklären: Trotz osteosynthetischer Versorgung entwickeln 38\% der Patienten eine posttraumatische Arthrose. Ihr Hauptrisikofaktor bleibt jedoch die nicht anatomische Rekonstruktion $[1,5]$.

\section{Revisionsoperation}

\section{Anspruchsvolle Ausgangslage}

Aufgrund der vorangegangenen Operationen und verletzungsbedingten Veränderungen der Weichteile besteht ein deutlich erhöhtes Infektionsrisiko für weitere Folgeeingriffe. Zudem erschweren v. a. pseudarthrotisch verheilte Frakturen und Knochennekrosen mit Defektsituation weitere Osteosynthesen und können knöcherne Resektionen erforderlich machen. Mit Stufenbildung verheilte Frakturen stellen ebenfalls ein operationstechnisches Problem dar. Inwieweit eine Reosteotomie eine Reduktion der Stufe ermöglicht oder ob diese vor dem Hintergrund einer späteren endoprothetischen Versorgung belassen werden kann, muss individuell entschieden werden. Aufgrund der knöchernen Veränderungen und Defekte sind oft Spezialimplantate erforderlich.

Nicht zuletzt auch wegen des erhöhten Operationsrisikos mit teilweise erheblichen Blutverlusten sollten diese Revisionsoperationen Zentrumskrankenhäusern mit entsprechendem peri- und postoperativen Management vorbehalten bleiben. Die Ausgangslage ist daher bei weitem nicht mit der bei primärer Koxarthrose vergleichbar. 


\section{Zeitpunkt}

Notfallindikationen bzw. dringliche Operationsindikationen bestehen bei infizierten Wunden, die einer unmittelbaren operativen Sanierung bedürfen.

Revisionsoperationen der Osteosynthese können zu unterschiedlichen Zeitpunkten sinnvoll sein. Für jeden Patienten ist daher eine individuelle Therapiestrategie festzulegen.

Bereits während der primären Osteosynthese sind die Stellung der Fragmente auf Stufenfreiheit zu überprüfen und jede kritische Schraubenlage zu ändern. Nur durch eine gewissenhafte Osteosynthese mit durch Sicht oder 3D-Bildwandler kontrollierten Schrauben lassen sich unnötige Revisionseingriffe verhindern. Bei Vorliegen intraartikulärer Schrauben (- Abb. 2) sollte unmittelbar die Indikation für eine Korrektur gestellt und nicht die Zerstörung des Gelenks abgewartet werden. Gegebenenfalls ist eine Verlegung des Patienten in ein entsprechendes Traumazentrum zu erwägen, da Revisionseingriffe komplikationsträchtig sind und eine komplexe intraoperative Bildgebung erforderlich machen können.

Auch bei verbliebenen zentralen Defekten (- Abb.3) ist eine Revisionsoperation frühzeitig anzustreben, denn eine stufenfreie Stellung nach fehlverheilter Fraktur lässt sich durch Reosteotomien nur noch schwer erreichen. Zudem besteht bei frühzeitiger Korrektur die Möglichkeit, den Defekt unmittelbar zu unterfüttern. - Abb. 3 zeigt einen nach initialer Osteosynthese verbliebenen zentralen Defekt (- Abb. 3a), bei welchem nach frühzeitiger operativer Revision eine anatomische Rekonstruktion des Pfannendaches erreicht werden konnte ( $\mathbf{\square}$ Abb. 3b).

Eine insuffiziente Osteosynthese mit ausgebrochenen Schrauben oder größerer Spaltbildung ( $\bullet$ Abb.4) sollte ebenfalls frühzeitig korrigiert werden. Ziel ist auch hier eine stufenfreie Gelenkfläche, um die drohende posttraumatische Arthrose zu verhindern. Bei ausgeprägten Defekten sollte vor dem Hintergrund einer späteren endoprothetischen Versorgung ein möglichst belastbarer Pfannengrund erzeugt werden. Daher können bei deutlicher Spaltbildung Revisionen gerechtfertigt sein, um durch besse-

Trauma Berufskrankh 2012 · 14[Suppl 2]:135-139 DOI 10.1007/s10039-011-1793-1

(c) Springer-Verlag 2011

\section{Jacobs · P.A. Grützner}

\section{Revisionseingriffe nach Azetabulumfraktur. Was macht Sinn?}

\section{Zusammenfassung}

Bei operativ versorgten Azetabulumfrakturen sind Komplikationen und langwierige Verläufe nicht selten. Ziel jeder Osteosynthese sollte eine anatomiegerechte Rekonstruktion mit stufenfreier Wiederherstellung der Gelenkflächen sein. Bei verbliebenen Stufen oder insuffizienten Osteosynthesen empfiehlt sich eine frühzeitige Revision. Bei posttraumatischer Arthrose ist meist eine Endoprothesenimplantation indiziert. Bei jüngeren Patienten ist ein stabiles Pfannenlager von Bedeutung, da im Verlauf ein Wechsel der Komponenten zu erwarten ist. Bei älteren Patienten kann bei 1-Pfeiler-Frakturen eine primäre Prothesenimplantation erwogen werden. Bei 2-Pfeiler-Frakturen sollte die Konsolidierung des Pfannengrunds abgewartet werden, da ansonsten eine Verankerung der Pfannenkomponente nicht gewährleistet ist. En- doprothesen nach vorheriger Osteosynthese sind mit Ergebnissen nach einem endoprothetischen Revisionseingriff vergleichbar. Die posttraumatische Endoprothese weist schlechtere Ergebnisse als die primäre Endoprothese bei Arthrose auf. Die Patienten profitieren jedoch von der verbesserten Funktionalität gegenüber einer Girdlestone-Situation oder gar einer Hüftgelenkarthrodese. Auch in der Hand des Erfahrenen ist die Versorgung der posttraumatischen Koxarthrose eine Herausforderung und setzt eine individuelle Planung voraus.

\section{Schlüsselwörter}

Azetabulumverletzungen .

Revisionsoperation · Koxarthrose ·

Endoprothese - Postoperative Komplikation

\section{Revision surgery following acetabular fractures. What makes sense?}

\begin{abstract}
Complications and protracted courses are not uncommon following acetabular fractures. The aim of osteosynthesis should always be anatomical reconstruction with a step-free restoration of joint areas. If steps remain or osteosynthesis is insufficient, early revision is recommended to achieve optimal results. In the case of posttraumatic arthrosis, endoprosthesis can often not be avoided. A stable cup position is important in younger patients, since a change of endoprosthetic components is to be expected. For older pa-

prosthetic components cannot be achieved. Endoprosthesis after previous osteosynthesis is comparable with results seen in endoprosthetic revision surgery. Post-traumatic endoprosthesis has worse results than primary endoprosthesis in cases of osteoarthritis. Nevertheless, patients benefit from the better functionality compared to a Girdlestone situation or even a hip joint arthrodesis. The management of post-traumatic coxarthrosis is a challenge even in experienced hands and requires individual planning.
\end{abstract} tients primary hip prosthesis can be considered in the case of single-column fractures. In the case of two-column fractures, consolidation of the cup fundament should first be awaited; otherwise, anchorage of the endo-

\section{Keywords}

Acetabulum, injuries · Revision surgery . Osteoarthritis, hip · Hip prosthesis . Complication, postoperative ren Knochenkontakt eine Pseudarthrose zu verhindern.

Bei verzögerten Revisionseingriffen mit bereits eingetretener Knochenbruchheilung ist als Gelenk erhaltendes Verfahren eine Osteotomie in Betracht zu ziehen. Bei einer Fehlstellung des Pfannendachs mit mangelhafter Überdachung des Femurkopfs kann beim jungen Patienten eine Osteotomie mit Pfannendachplas- tik zu einer Optimierung der Lastverteilung führen. Ziel ist es, eine Endoprothese so lang wie möglich hinauszuschieben, um auch eine Wechseloperation im Lauf des Patientenlebens zu vermeiden. Auf der anderen Seite muss jedoch auch bedacht werden, dass der Patient bei unproblematischer primärer Prothesenimplantation von einer zügigen Steigerung der Lebensqualität profitiert und ihm frustra- 


\section{Becken, Acetabulum, hüftgelenksnahe Frakturen}

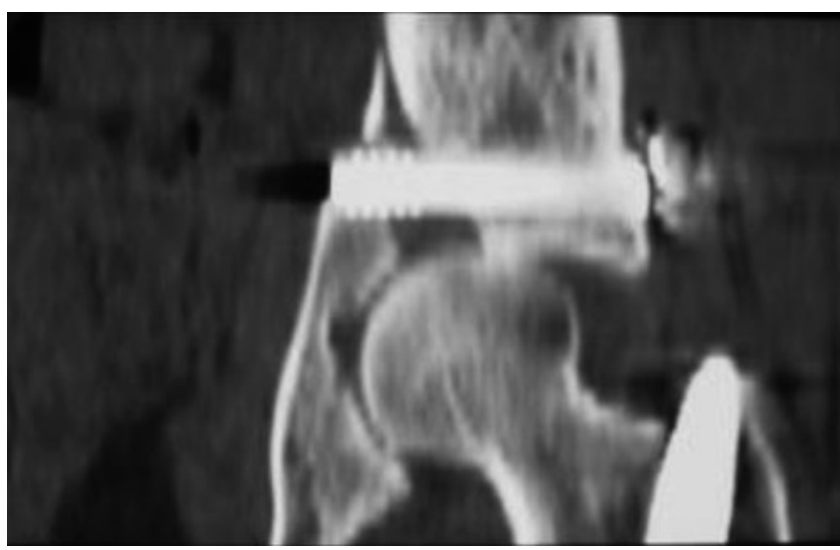

Abb. $4<$ Insuffiziente Primärstabilisation

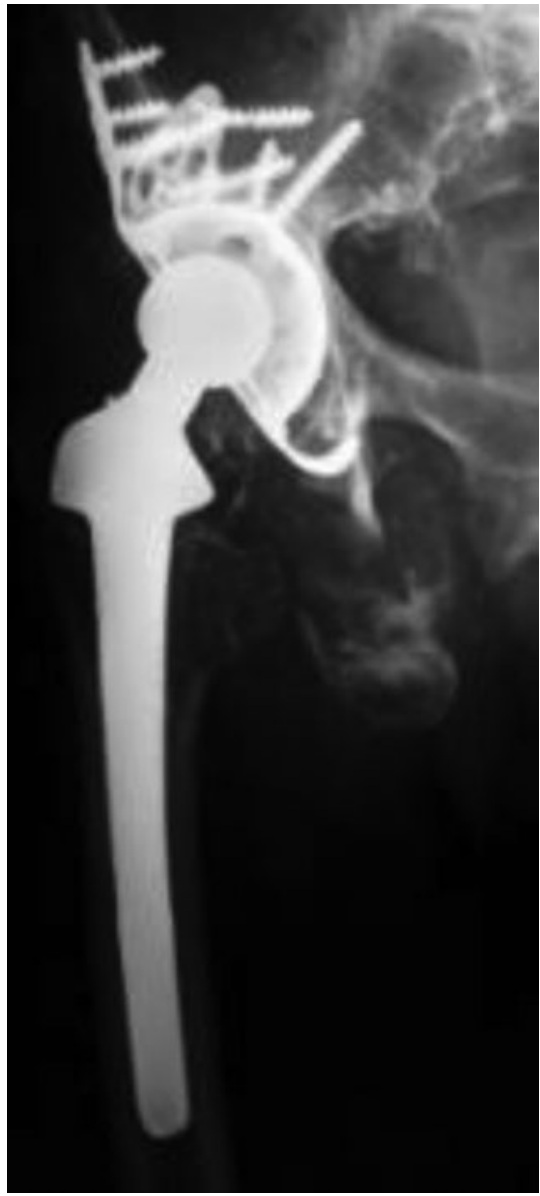

Abb. $5 \Delta$ Pfannenaufbauplastik mit Spezialimplantaten nach Azetabulumfraktur

ne Osteotomien mit langen Entlastungsphasen erspart bleiben. Auch hier ist daher die Entscheidung immer in Abhängigkeit vom Alter, Aktivitätsgrad und Anspruch des Patienten zu treffen.

\section{Endoprothese}

Häufig lässt sich die posttraumatische Arthrose nicht langzeitig verhindern, sodass früher oder später die Indikation für eine Endoprothese diskutiert werden muss. Im eigenen Patientengut waren bei 45 prospektiv untersuchten Patienten (19992006) endoprothetische Versorgungen 8,8 Jahre nach primärer Osteosynthese erforderlich (3 Monate bis 50 Jahre). Bei 18 von 45 lag eine Femurkopfnekrose vor [6].

Prothesenimplantationen nach operativ versorgten Azetabulumfrakturen sind anspruchsvolle Operationen mit einem hohen Risiko an Komplikationen und sollten nicht zuletzt auch wegen der oft erforderlichen Spezialimplantate Traumazentren vorbehalten bleiben.

In - Abb. 5 ist eine komplexe Pfannenaufbauplastik mit Spezialimplantaten zur Verankerung der Pfannenkomponente dargestellt.

Vor der endoprothetischen Versorgung ist ggf. die Metallentfernung der Osteosynthese zu planen. Schrauben und Platten sollten entfernt werden, wenn ein Kontakt mit der zu implantierenden Pfanne zu erwarten ist. Zu berücksichtigen ist dabei auch, dass die geplante Pfannenposition durch Fräsen vertieft wird und sich die Distanz zur Osteosynthese vermindert. In unserer Klinik gilt als Untergrenze des zu tolerierenden Abstands eine Dis$\tan z$ von $1 \mathrm{~cm}$ zum Osteosynthesematerial.

Bei der Wahl des Zugangswegs muss bedacht werden, dass dorsal liegende Im-

\section{Metallentfernung vor Endoprothese}

plantate über einen lateralen Zugang nur schwer entfernbar sind. In diesem Fall empfiehlt sich ein zweizeitiges Vorgehen über einen gesonderten Zugang oder die Prothesenimplantation über den dorsalen Zugang. Vor der endgültigen Prothesenimplantation ist eine Punktion des Hüftgelenks zum Ausschluss eines „low grade“Infekts durchzuführen.

Aufgrund der knöchernen Defekte sind häufig Spongiosatransplantationen oder die Implantation von Fremdknochen erforderlich. Generelle Behandlungsalgorithmen lassen sich hierfür nicht formulieren, da zahlreiche Faktoren die Operationstechnik mit beeinflussen und berücksichtigt werden müssen. Dennoch gilt, dass in knöchernen Defekten keine Implantate belassen werden sollten, denn sie können in nachfolgenden Operationen eine Defektauffüllung verhindern und somit die spätere Pfannenverankerung erschweren.

\section{Patientenalter}

Das Alter und der individuelle Mobilitätsgrad des Patienten müssen berücksichtigt werden. Bei jüngeren Patienten steht eine lange Haltbarkeit der endoprothetischen Versorgung im Vordergrund. Auch vor dem Hintergrund noch erforderlicher Wechseloperationen ist ein gutes knöchernes Fundament für eine sichere Verankerung von entscheidender Bedeutung. Knöcherne Pfannendefekte können mit autologer Spongiosa oder Fremdknochen aufgebaut werden. Wenn möglich sollten zementfreie Implantate zur Anwendung kommen. Im Anschluss an die Operation sind ggf. Entlastungsphasen notwendig, um eine Integration der Prothese zu gewährleisten.

14\% (118 von 823) der Beckenfrakturen betreffen Patienten $>65$ Jahre [2]. Ältere Patienten können von der frühen Belastungsfähigkeit zementierter Implantate profitieren. Es besteht häufig unmittelbar Vollbelastbarkeit, da keine Integration der Prothese abgewartet werden muss. Zudem benötigt eine zementfreie Implantation für einen verlässlichen Halt ein stabiles Knochenlager, welches bei osteoporotischem Knochen problematisch sein kann. Die Rekonstruktionsmöglichkeiten der knöchernen Defekte sind limi- 
tiert und setzten ein gutes Heilungspotenzial voraus. Daher können sich aufwendige Rekonstruktionen bei älteren Patienten allein durch eine schlechtere Knochenqualität und begleitende Komorbiditäten verbieten. Oft muss bei größeren Defekten auf Spezialimplantate der Revisionsendoprothetik zurückgegriffen werden.

Während bei jüngeren Patienten der Erhalt des eigenen Gelenks im Vordergrund und im Fokus eine möglichst anatomische Rekonstruktion stehen, ist bei älteren Patienten auch eine primär endoprothetische Versorgung zu diskutieren. Vor allem bei 1-Pfeiler-Frakturen kann eine gute Verankerung der Pfannenkomponente gelingen und erspart dem Patienten lange Entlastungsphasen nach einer Osteosynthese. Bei immerhin 36-64\% der älteren Patienten (>65 Jahre) bestehen solche isolierten Frakturen des vorderen Pfeilers [2]. Bei 2-Pfeiler-Frakturen dagegen ist in der Regel unabhängig vom Patientenalter eine primäre Osteosynthese zur Rekonstruktion der Pfanne das sinnvollere Therapiekonzept.

\section{Fazit für die Praxis}

- Ziel einer jeden Osteosynthese sollte eine anatomiegerechte Rekonstruktion mit stufenfreier Wiederherstellung der Gelenkflächen sein (Spalt $<2 \mathrm{~mm}$ ).

- Bei größeren Stufen oder intraartikulären Schrauben ist eine frühzeitige Revision anzustreben, um ein optimales Resultat zu erzielen.

- Bei 1-Pfeiler-Frakturen älterer Patienten ist die primäre Prothesenimplantation eine Option.

- Bei 2-Pfeiler-Frakturen sollte unabhängig vom Patientenalter die Konsolidierung des Pfannengrunds abgewartet werden, da eine Verankerung der Pfannenkomponente sonst nicht gewährleistet ist.

- Bei Vorliegen einer posttraumatischen Arthrose ist die Endoprothese nicht zu umgehen.

- Vor derselben ist eine Metallentfernung erforderlich, wenn die zu erwartende Distanz unter $1 \mathrm{~cm}$ beträgt.

- Jüngere Patienten benötigen ein stabiles Pfannenlager v. a. vor dem Hin- tergrund noch erforderlicher Wechseloperationen.

- Bei älteren Patienten sind zementiere Implantate gerechtfertigt.

- Endoprothesen nach vorheriger Osteosynthese sind mit einem endoprothetischen Revisionseingriff vergleichbar und führen zu schlechteren Ergebnissen als die primäre Endoprothese bei Arthrose.

- Auch in der Hand des Erfahrenen ist die Versorgung der posttraumatischen Koxarthrose eine Herausforderung und setzt eine individuelle Planung, Spezialimplantate und intraoperative Bildgebung voraus.

\section{Korrespondenzadresse

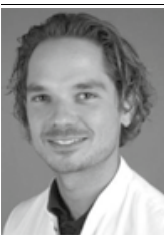 \\ Dr. C. Jacobs \\ Klinik für Unfallchirurgie und Orthopädie, BG-Unfallklinik Ludwigshafen, Ludwig-Guttmann-Straße 13, 67071 Ludwigshafen cjacobs@bgu-ludwigshafen.de}

Interessenkonflikt. Der korrespondierende Autor gibt an, dass kein Interessenkonflikt besteht.

\section{Literatur}

1. Briffa N, Pearce R, Hill AM, Bircher M (2011) Outcomes of acetabular fracture fixation with ten years follow-up. J Bone Joint Surg Br 93(2):229236

2. Ferguson TA, Patel R, Bandari M, Matta JM (2010) Fractures of the acetabulum in patients aged 60 years and older. J Bone Joint Surg Br 92-B:250257

3. Gänsslen A, Steinke B, Krettek C (2009) Osteosynthese von Frakturen der hinteren Wand des Azetabulums. Oper Orthop Traumatol 21:283-295

4. Porter SE, Russell GV, Dews RC et al (2008) Complications of acetabular fracture surgery in morbidly obese patients. J Orthop Trauma 22:589-594

5. Ragnarsson B, Mjöberg B (1992) Arthrosis after surgically treated acetabular fractures. A retrospective study of 60 cases. Acta Orthop Scand 63(5):511-514

6. Schulte-Bockholt D, Frank C, Wentzensen A (2007) Besonderheiten der endoprothetischen Versorgung bei der posttraumatischen Coxarthrose nach Azetabulumfraktur. In: Weller S, Braun A, Eingartner $\mathrm{CH}$ et al (Hrsg) Das Bicontact Hüftendoprothesensystem 1987-2007. Thieme, Stuttgart New York, S 116-122 (Kapitel 4)

7. Vallier HA, Cureton BA, Ekstein C et al (2010) Early definitive stabilization of unstable pelvis and acetabulum fractures reduces morbidity. J Trauma 69(3):677-684 